$10 / 26-95800$

UCRL-ID-119916

\title{
Scaling Laws for Specialized Hohlraums
}

\author{
Mordecai D. Rosen
}

ICF Target Physics Program

Lawrence Livermore National Laboratory

Livermore, Ca 94550

September 28, 1993

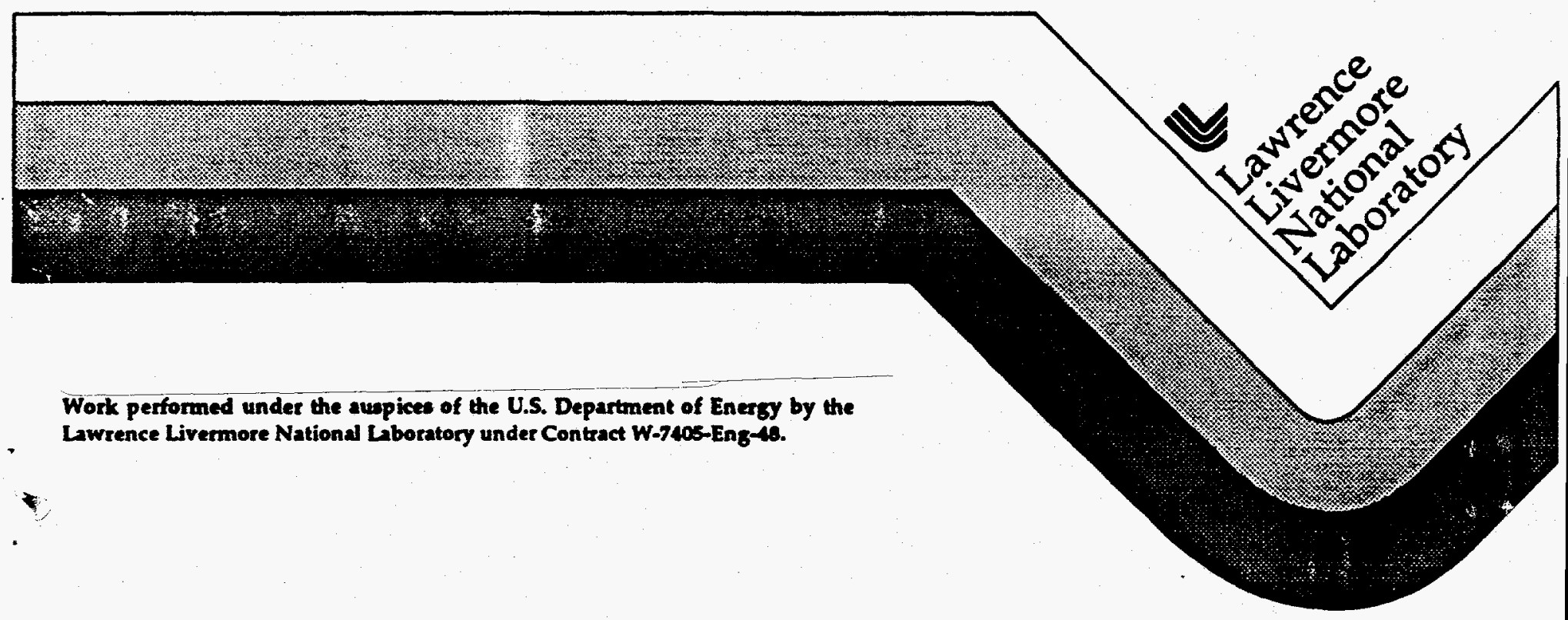

Work performed under the aupices of the U.S. Department of Energy by the

Lawrence Livermore National Laboratory under Contract W-7405-Eng-40. 


\section{DISCLAIMER}

This document was prepared as an account of work sponsored by an agency of the United States Goverameat Neither the United States Goverument nor the University of Callforaia mor any of their employees, makes any warranty, express or bmplied, or ascumes any legal tiability or respensibility for the aceuracy, completeness, or usefulaess of any information, apparatus, product, or process disclosed, or represents that its we would wot intringe privately ormed rights. Reference hercia to any specific commercial products, process, or service by trade anme, tradeanark, manufscturer, or otherwise, does not necessarily constitute or imply its endorsement recommendation, or favoring by the Uniled States Government or the Univerdity or Callforain. The viewsand opinions of authors expressed herein do nol necessarily state or reflect those of the United States Governinent or the University of Calffornia, and shall not be used for advertising or product endorsement purposes.

This report has been reproduced directly from the best available copy.

Available to DOE and DOE contractors from the Ofisce of Scientific and Technical Information P.O. Box 62, Oak Ridge, TN 37831

Prices available from (615) 576-8401, FTS $626-8401$

Avallable to the public from the

National Technical Information Service

US. Department of Commerce 5285 Port Rojal Rd.

Springfield, VA 22161 


\section{DISCLAIMER}

Portions of this document may be illegible in electronic image products. Images are produced from the best available original document. 
Scaling Laws for Specialized Hohlraums

\author{
Mordecai D. Rosen \\ ICF Target Physics Program \\ Lawrence Livermore National Laboratory \\ Livermore, CA 94550
}

September 28, 1993 


\title{
Scaling Laws for Specialized Hohlraums
}

\author{
Mordecai D. Rosen \\ ICF Target Physics Program \\ Lawrence Livermore National Laboratory \\ Livermore, CA 94550
}

\begin{abstract}
We present scaling laws for the behavior of hohlraums that are somewhat more complex than a simple sphere or cylinder. In particular we consider hohlraums that are in what has become known as a "primary" "secondary" configuration, namely geometries in which the laser is absorbed in a primary region of a hohlraum, and only radiation energy is transported to a secondary part of the hohlraum that is shielded from seeing the laser light directly. Such hohlraums have been in use of late for doing LTE opacity experiments on a sample in the secondary and in recently proposed "shimmed" hohlraums that use gold disks on axis to block a capsule's view of the cold laser entrance hole. The temperature/drive of the secondary, derived herein, scales somewhat differently than the drive in simple hohlraums.
\end{abstract}

\section{Introduction}

In a companion paper ${ }^{1}$ in these proceedings we have rederived the basic scaling laws for radiation drive in simple laser driven hohlraums. It is our purpose in this paper to extend those results and methodologies to more complicated hohlraum geometries. In particular we consider hohlraums that are in what has become known as a "primary" " secondary" configuration, namely geometries in which the laser is absorbed in a primary region of a hohlraum, and only radiation energy is transported to a secondary part of the hohlraum that is shielded from seeing the laser light directly. Such hohlraums have been in use of late for doing LTE opacity experiments on a sample in the secondary. Such hohlraums have come under the jargon titles of "hohlraums with lips" and "McFee hohlraums". The laser beams enter through holes in the end caps of a cylinder, are absorbed along the walls, and along "lips" that protrude perpendicularly from the cylinder walls, in regions near the end caps. Radiation flows from this primary region through the circular aperture defined by the lips, into the secondary region, namely the central region of the cylinder which holds a capsule or sample. These geometries have been in use for a long time. A decade or two ago they were used on two beam facilities such as Argus and Novette, wherein the incident laser struck a scattering cone in the 
primary of a cylindrical hohlraum, the capsule sat in the center of the secondary, and radiation flowed to it by passing through the annular region between the on axis scattering cone and the cylinder walls.

History aside, the temperature/drive of the secondary, derived herein, scales somewhat differently than the drive in simple hohlraums. McFee and Wilde ${ }^{2}$ have presented scaling laws for the secondary drive temperature derived empirically from LASNEX simulations. They find that at constant input laser energy $E_{L}$, the drive $T$ scales as $s^{-3 / 4}$ where $s$ is a scale size. At constant $s$ they find $T$ scales as $E^{1 / 2.7}$. (Note to the reader: in this paper we will be using the symbols $E$ and $E_{L}$ fairly interchangeably ). Their paper (or at least early to recent versions of it) try to motivate the $s^{-3 / 4}$ scaling in terms of radiation energy scaling as volume times $\mathrm{T}^{4}$, or $\mathrm{s}^{3} \mathrm{~T}^{4}$. Therefore, at constant $\mathrm{E}, \mathrm{T}$ scaling as $\mathrm{s}^{-3 / 4}$ would clearly ensue. We find two difficulties with this explanation. First, while it is true that the energy in a radiation field does scale that way, in these systems of interest, the amount of energy in the radiation field is small, only a percent or two of the total available enrgy - the bulk of the energy resides in the Au walls of the hohlraums. Thus holding laser energy fixed should not be equated with holding the energy in the radiation field fixed. Secondly the scaling of $E$ as $s^{3} T^{4}$ would imply that at constant $s, T$ should scale as $E^{1 / 4}$ in contradiction to the $E^{1 / 2.7}$ that is observed in their simulations.

In part II of this paper we will derive their observed scaling. While a very quick derivation can be given in one line, we find that it too is over simplified. The quick derivation is simply to say that $\mathrm{E}$ scales as $s^{2} \mathrm{~T}^{2.7}$. This already has many virtues. Namely it scales as wall area, $\mathrm{s}^{2}$, not hohlraum volume, $\mathrm{s}^{3}$, as is appropriate for our situation in which almost all the energy resides in the wall. The wall loss scaling of $\mathrm{T}^{2.7}$ is not that dissimilar to the $\mathrm{T}^{3.2}$ derived in our companion paper ${ }^{1}$ for simple hohlraums. In one fell swoop this scaling reproduces the simulation results. At constant $E, T$ should scale as $s^{-2 / 2.7}$ or $s^{-0.74}$. At constant $\mathrm{s}, \mathrm{T}$ should scale as $\mathrm{E}^{1 / 2.7}$. Thus both observations are derived by this simple formula. There are, however, two flaws in this argument. First, it is an oversimplification to use results for simple hohlraums and apply them to these primary/secondary geometries. Secondly, the $T^{2.7}$ vs. $T^{3.2}$ makes one wary as well. In the ensuing section we will solve the scaling problem more properly, accounting for the 
geometry, and find full consistency with our previous results, and with the simulation results of McFee and Wilde as well.

In part III of this paper we will apply our methodology to yet another primary secondary configuration, namely "shimmed hohiraums" presented by Amendt and Murphy in these procedings ${ }^{3}$. Here a shim disk on axis shields the capsule from seeing the cold laser entrance hole. In this case, we are presented with the curious configuration of an inside out McFee hohlraum, in which the primary is essentially the central section of the cylinder which holds the capsule.The laser beams enter the cylinder through entrance holes in the end cap as usual, but propagate through the outer "secondary" section, pass between the shim disk on axis and the wall, and impinge on the walls in the central section of the hohlraum. Thus the end sections of the cylinder are the cooler secondary, and the aperture through which the radiation flows from the middle (primary) to the ends (secondaries) is the annulus between the on axis shim disk and the cylinder wall. 


\section{Primary / Secondary Scaling}

In primary/secondary hohlraums, laser light enters the hohlraum interior through laser entrance holes located in either end cap of the cylinder. The light is absorbed at the cylinder walls in the primary region of the cylinder, converting laser light into soft $\mathrm{x}$-rays. These $\mathrm{x}$-rays are rapidly absorbed and reemitted by the walls setting up a radiation driven thermal wave diffusing into the walls (a so called "Marshak Wave"). Some of the $x$-rays escape out the laser entrance holes (LEH) while others flow through the primary secondary gap into the secondary region of the hohlraum. There they are absorbed by the walls in the secondary section of the cylinder and by whatever sample there may be there. We will ignore the sample here, as it is typically a small energy sink.

In our companion paper ${ }^{1}$, the basic scaling of hohlraum wall loss due to the Marshak Wave was derived. We found the wall loss to scale as $\mathrm{T}^{3.2}$ for XSN opacity and as $\mathrm{T}^{3.4}$ for STA opacity. As we are uncertain of exactly what opacity was used by McFee and Wilde, with the presumption that it was quite close to ours, for arbitrariness sake we will split the difference here and postulate E scaling as $\mathrm{T}^{\mathbf{3 . 3}}$. Clearly, the small differences here are not crucial for the derivation that will follow.

The energy balance in the primary can be written as:

$$
\eta E_{L}=E_{W P}+E_{L E H}+E_{P S}
$$

where the lhs of the equation represents the source of $x$-rays coming from the conversion of laser energy $E_{L}$ into $x$-rays with an efficiency $\eta$. The right hand side represents the three sinks of energy in the primary; EWP is the wall loss in the primary, ELEH is the laser entry hole loss, and EPS is the net loss of of radiant energy flowing from the primary to the secondary. We will consider only 1 nsec flat top laser drive pulses here for simplicity, and thus ignore time dependence completely in the scaling arguments presented below, for ease of presentation. Clearly the time dependence, which was the major focus of our companion paper $^{1}$ could be put in for more generality. As discussed above, we take EWP to scale as $\mathrm{TP}^{3.3}$ AP. where 
AP is the primary wall area and $T_{P}$ is the primary radiation temperature. ELEH clearly scales as $T_{P}{ }^{4} A_{H}$ where AH is the LEH area. EPS scales as APS $\left(T_{P}^{4}-T_{S}^{4}\right)$ where APS is the area of the gap between the primary and the secondary through which the radiation flows, and TS is the secondary temperature.

The energy balance in the secondary can be written as:

$$
\text { EPS }=\text { EWS }
$$

where EPS has already been defined, and in the secondary serves as the source of radiation. The sink is EwS the wall loss in the secondary, which scales as $\mathrm{TS}^{3.3} \mathrm{AS}$, in which AS is the secondary wall area. Let us define $\mathrm{y}$ as $\mathrm{TS} / \mathrm{TP}$, combine Eq. (1) and (2) to obtain Eq. (3), and rewrite Eq.(2) as Eq (4):

$$
\begin{aligned}
& \eta E_{L}=c \text { AP } \operatorname{TP}^{3.3}\left(1+\varepsilon \operatorname{TP}^{0.7}+\delta y^{3.3}\right) \\
& \text { APS } \operatorname{TP}^{4}\left(1-y^{4}\right)=c \delta A_{P} \operatorname{TP}^{3.3} y^{3.3}
\end{aligned}
$$

where the constant $c$ represents the wall loss coefficient, and $\varepsilon$ and $\delta$ represent ratios of the areas AH/AP and AS/AP respectively. We anticipate that $y$ will be less than one and thus will ignore $y^{4}$ compared to 1 and $\delta y^{3.3}$ compared to 1 . Assuming $\varepsilon$ is small (it usually is), and for the purposes of simplifying even further, we drop that term as well, leading Eq (3) to reduce to:

$$
E=s^{2} T p^{3.3}
$$

where we have ignored consants for now and note that the area AP scales as scale size s, squared. In Eq. (4) the areas that appear on both sides of the equation cancel the s scaling, and we have the simple equation:

$$
\mathrm{TP}^{0.7}=\mathrm{y}^{3.3}
$$

again ignoring constants for now. Thus Eq. (6) implies that

$$
y=T_{P} 0.21
$$

which means that 


$$
T_{S}=y \quad T P=T P 1.21
$$

Using Eq. (5) we obtain

$$
\mathrm{TS}=\left(\mathrm{E} / \mathrm{s}^{2}\right)^{1.21 / 3.3}=\left(\mathrm{E} / \mathrm{s}^{2}\right)^{1 / 2.7}
$$

This is the central result of this analysis, and is precisely the form required to fit the two observations based on the LASNEX simulations of McFee and Wilde, namely that at fixed E, T scales as $\mathrm{s}^{-.74}$, and that at fixed $s, T$ scales as $E^{1 / 2.7}$. Moreover, it is completely consistent (indeeed, derived on the basis of) the scaling laws for wall loss derived in our previous paper, which dealt with simple geometry hohlraums.

Had we kept track of constants, coefficients etc. we could have quantitatively shown that these sets of equations lead to the simulation results. For example a scale $2 \mathrm{McFee}$ hohlraum on Nova would, on the basis of these equations, get a primary temperature of about $195 \mathrm{eV}$ and a secondary temperature of about $115 \mathrm{eV}$, in reasonable agreement with the LASNEX simulations. For brevity sake we omit those details here, but in the next section will give a detailed numerical example of these equations in action. 


\section{Shimmed Hohlraums}

We now apply our methodology to yet another primary secondary configuration, namely "shimmed hohlraums" presented by Amendt and Murphy in these procedings ${ }^{3}$. The laser beams enter the cylinder through entrance holes in the end cap as usual, but propagate through the outer "secondary" section, into the central section of the hohlraum, and impinge on the walls there. The outer parts are the cooler secondary, and the aperture through which the radiation flows from the middle (primary) to the ends (secondaries) is the annulus between the on axis shim disk and the cylinder wall.

The LASNEX "observables" to be explained here are the $230 \mathrm{eV}$ for a hohlraum with no shims vs. a $240 \mathrm{eV}$ drive on capsule for one with shims. Why would a hohlraum that introduces about $500 \mathrm{~J}$ of more wall loss via the shim disks, actually produce a hotter hohlraum rather than a cooler one? The answer to the paradox is essentially that we have created an inside out McFee hohlraum, in which the central section is the hotter primary, and drives the capsule. Indeed, the outer sections of these hohlraums are the cooler secondaries, and are predicted by LASNEX to be only about $215 \mathrm{eV}$. We will derive all of these numbers presently.

The cylinder is $0.8 \mathrm{~mm}$ in radius with a laser entrance hole (LEH) of $0.6 \mathrm{~mm}$ radius. The half length (from mid plane to end cap) of the can is $1.15 \mathrm{~mm}$. The shim is $0.325 \mathrm{~mm}$ in radius and placed on axis $0.65 \mathrm{~mm}$ from the midplane, (thus, $0.5 \mathrm{~mm}$ from the $\mathrm{LEH}$ ). The capsule has a radius of $0.275 \mathrm{~mm}$. Thus the half-area of the primary walls (from midplane to shim and including the shim disk area) AP is 3.6 $\mathrm{mm}^{2}$, of the secondary $A_{S} 3.7 \mathrm{~mm}^{2}$ and of the half-capsule $A_{C} 0.47 \mathrm{~mm}^{2}$. The $\mathrm{LEH}$ area $A_{L}$ is $0.9 \mathrm{~mm}^{2}$, and the annular area between shim and wall, APS, through which the radiation flows from primary to secondary is $1.7 \mathrm{~mm}^{2}$. A slight complication here is that some (about 20\%) of the laser energy is deposited along the cylinder wall between the axial position of the shim and the end of the can; namely there is some of the laser source in the secondary region. Nonetheless we can generalize our treatment in Sec. II to account for this situation, and we will define $E_{\mathrm{L}}$ to be made up of two parts $E_{\mathrm{LP}}$ and $\mathrm{E}_{\mathrm{LS}}$ for those 
amounts absorbed in the primary and secondary regions respectively. In our case, for half of the incident $20 \mathrm{KJ}(=200 \mathrm{~h}$ ) going into the half of the hohlraum being calculated, and at a conversiion efficiency of $70 \%$, there is $1 / 2$ of $140 \mathrm{hJ}$ of xray energy available. If $20 \%$ is created in the secondary, then $56 \mathrm{hJ}$ is available in the primary and $14 \mathrm{hJ}$ in the secondary. Using the same notations of section II (in particular, $\mathrm{y}=$ $\mathrm{TS}_{\mathrm{S}} \mathrm{TP}_{\mathrm{P}}$ ) and appealing as we did there to energy balance, and using the XSN scaling laws for wall loss, hole loss, capsule loss("EC") etc. as presented in Ref 1, (for a 1 nsec flat top pulse) we find the following:

The primary equation reads:

$$
\begin{array}{r}
\eta E_{L P}=\quad E_{W P}+E_{P S}+E_{C} \quad \text { or } \\
\eta E_{L P}=.44 T_{P}{ }^{3.2} A_{P}+.7 \text { APS }_{P} P^{4}\left(1-y^{4}\right)+.7 A_{C} T_{P}{ }^{4}
\end{array}
$$

while the secondary equation reads:

$$
\begin{aligned}
& \eta E_{L S}+. \quad \text { EPS }=\text { EWS }+E_{L E H} \text { or } \\
& \eta E_{L S}+.7 \operatorname{APST}^{4}\left(1-y^{4}\right)=.44 \mathrm{TP}^{3.2} \mathrm{y}^{3.2} \mathrm{AS}_{\mathrm{S}}+.7 \mathrm{ALTP}^{4} \mathrm{y}^{4}
\end{aligned}
$$

Using Eq. (11) in Eq. (10) we rewrite Eq. (10) as:

$$
\eta E_{L}=\eta\left(E_{L P}+E_{L S}\right)=.44 \mathrm{TP}^{3.2} \mathrm{AP}\left(1+\left(\mathrm{AS}_{\mathrm{S}} / \mathrm{AP}\right) \mathrm{y}^{3.2}\right)+.7 \mathrm{ACTP}^{4}\left(1+\left(\mathrm{A}_{\mathrm{L}} \mathrm{AC}_{\mathrm{C}} \mathrm{y}^{4}\right)\right.
$$

Inserting the values for the areas (discussed above) Eq. (12) reduces to:

$$
70=1.6 \mathrm{Tp}^{3.2}\left(1+\mathrm{y}^{3.2}\right)+.33 \mathrm{Tp}^{4}\left(1+1.9 \mathrm{y}^{4}\right)
$$

while Eq. (14) reduces to:

$$
14+1.2 T_{P^{4}}\left(1-y^{4}\right)=1.6 T_{P}^{3.2} y^{3.2}+.64 T_{P}^{4} y^{4}
$$

Equations (13) and (14) can be solved iteratively with a solution quickly converging to $T_{p}=2.45$ and $y=$ 0.88 , namely $T_{S}=y T_{p}=2.16$. As quoted above from Amendt's simulations, these values for the primary 
and secondary temperatures are in excellent agreement with LASNEX. Moreover, had we considered a simple geometry (no shims) we would be solving:

$$
\begin{aligned}
& \eta E_{L}= E_{W}+E_{L E H}+E_{C} \\
& \eta E_{L}=.44 T^{3.2} \mathrm{AW}+7 \mathrm{~T}^{4} \mathrm{~A}_{\mathrm{L}}+.7 \mathrm{ACT}^{4}
\end{aligned}
$$$$
\text { or }
$$

For the simple, no shim, geometry we find $A_{W}=6.7 \mathrm{~mm}^{2}$ and $A_{L}$ and $A_{C}$ are as above. Thus Eq. (15) reduces to:

$$
70=2.95 \mathrm{~T}^{3.2}+.96 \mathrm{~T}^{4}
$$

whose solution is $T=2.3$, again in excellent agreement with the LASNEX result for no shim of $228 \mathrm{eV}$.

\section{Summary}

We have seen that extending our hohlraum scaling laws from simple geometries to primary / secondary ones serves as a unifying treatment that can account for all of the results quoted thus far.

We gratefully acknowledge useful conversations with R. McFee, B. Wilde, and P. Amendt.

\section{REFERENCES}

${ }^{1}$ M.D. Rosen"Marshak Waves: Constant Flux vs. Constant $\mathbf{T}$ - a (slight) Paradigm Shift" these proceedings

2R. McFee and B. Wilde "Specialized Hohlraums" these proceedings

${ }^{3}$ T. Murphy and P. Amendt "Modified Hohlraums for Reducing Laser Entrance Hole Effects", these proceedings 